\section{MDC1 accelerates nonhomologous end-joining of dysfunctional telomeres}

\author{
Nadya Dimitrova and Titia de Lange ${ }^{1}$ \\ Laboratory for Cell Biology and Genetics, The Rockefeller \\ University, New York, New York 10021, USA
}

Here we document the role of MDC1 (mediator of DNA damage checkpoint 1 ) in the detection and repair of human and mouse telomeres rendered dysfunctional through inhibition of TRF2. Consistent with its role in promoting DNA damage foci, MDC1 knockdown affected the formation of telomere dysfunction-induced foci (TIFs), diminishing the accumulation of phosphorylated ATM, 53BP1, Nbs1, and to a lesser extent, $\gamma$-H2AX. In addition to this effect on TIFs, the rate of nonhomologous end-joining (NHEJ) of dysfunctional telomeres was significantly decreased when MDC1 itself or its recruitment to chromatin was inhibited. MDC1 appeared to promote a step in the NHEJ pathway after the removal of the 3 ' telomeric overhang. The acceleration of NHEJ was unlikely to be due to increased presence of 53BP1 and Mre11 in TIFs, since knockdown of neither factor affected telomere fusions. Furthermore, relevant cell cycle effectors (Chk2, p53, and p21) of the ATM kinase pathway were unaffected and there was no change in the rate of cell cycle progression. We propose that the binding of MDC1 to $\gamma-\mathrm{H} 2 \mathrm{AX}$ directly affects NHEJ in a manner that is independent of the ATM-dependent cell cycle arrest pathway.

Supplemental material is available at http://www.genesdev.org.

Received May 30, 2006; revised version accepted October 16, 2006

\section{Results and Discussion}

MDC1 (mediator of DNA damage checkpoint 1) is a recently identified mammalian DNA damage response factor with an important role in the DNA damage response (Goldberg et al. 2003; Stewart et al. 2003; Lukas et al. 2004; for review, see Stucki and Jackson 2006). MDC1 is recruited to chromatin near sites of DNA damage through the interaction of its BRCA1 C-terminal (BRCT) repeats with $\gamma$-H2AX, the phosphorylated form of $\mathrm{H} 2 \mathrm{AX}$ (Stewart et al. 2003; Lee et al. 2005; Stucki et al. 2005). Chromatin-bound MDC1 facilitates the recruitment of the ATM kinase to sites of DNA damage, leading to additional H2AX phosphorylation, which in turn recruits more MDC1 (Stucki et al. 2005; Bekker-Jensen et al. 2006; Lou et al. 2006). MDC1 also interacts with the

[Keywords: ATM; DNA damage; NHEJ; MDC1; telomere; TRF2] ${ }^{1}$ Corresponding author.

E-MAIL delange@mail.rockefeller.edu; FAX (212) 327-7147.

Article is online at http://www.genesdev.org/cgi/doi/10.1101/gad.1496606.
Mre11/Rad50/Nbs1 complex and promotes its stable association with sites of DNA damage (Goldberg et al. 2003), which could explain how MDC1 enhances the local action of the ATM kinase. Thus, MDC1 appears to be part of a feed-forward loop that promotes the stable association of DNA damage response factors in large domains (foci) near the site of damage (Stucki et al. 2005; Lou et al. 2006). However, the function of such DNA damage response foci, the role of the foci in the regulation of cell cycle progression, and their contribution to DNA repair have remained ill-defined (Stucki and Jackson 2006). Here we examine the contribution of MDC1 to the detection and processing of a specific form of DNA damage, chromosome ends lacking telomere protection. The results point to a direct role for MDC1 in the repair of dysfunctional telomeres through nonhomologous endjoining (NHEJ).

The protective function of telomeres can be disrupted through inhibition of the shelterin protein TRF2 with a dominant-negative allele or through deletion of the mouse TRF2 gene (for review, see de Lange 2005). Cells respond to the resulting unprotected chromosome ends as if they were double-strand breaks (DSBs). DNA damage factors such as 53BP1, $\gamma$-H2AX, ATM phosphorylated at S1981 (ATM-S1981-P), and the Mre11/Nbs1/Rad50 complex accumulate in telomere dysfunction-induced foci (TIFs) that extend from the dysfunctional telomere into the subtelomeric regions (d'Adda di Fagagna et al. 2003; Takai et al. 2003; Celli and de Lange 2005). Telomere damage also activates the ATM kinase signaling cascade, resulting in p53-mediated arrest before DNA replication, and the deprotected chromosome ends are processed by DNA repair pathways, resulting in telomere fusions. The pathway leading to telomere fusions was identified as NHEJ based on the 100-fold diminished fusion rate in the absence of DNA ligase IV /Celli and de Lange 2005) and the 10-fold reduction in cells lacking Ku70 (Celli et al. 2006). Thus, both the DNA damage signaling pathway and the DNA damage repair reactions elicited by TRF2 loss resemble the canonical response to DSBs. In diploid mammalian cells, TRF2 inhibition typically generates 10-90 $\gamma$-H2AX foci (Takai et al. 2003; Celli and de Lange 2005), which is a moderate insult equivalent to the number of DSBs resulting from irradiation with 0.25-2.5 Gy (Rothkamm and Lobrich 2003).

MDC1 was readily observed at telomeres when their function was compromised. Telomere deprotection was induced by Cre-mediated deletion of a conditional TRF2 allele (TRF2F/-) (Celli and de Lange 2005) from mouse embryo fibroblasts (MEFs). For these initial experiments, we used cells lacking DNA ligase IV (lig4) in order to block telomere fusions (Smogorzewska et al. 2002; Celli and de Lange 2005), and the MEFs also lacked a functional p53 pathway, abrogating their G1/S arrest (Smogorzewska and de Lange 2002). Upon deletion of TRF2 from such TRF2F/- lig4 ${ }^{-/-}$p53 ${ }^{-7-}$ cells, MDC1 formed large TIFs that coincided with 53BP1 TIFs (Fig. 1A). Similarly, MDC1 localized to TIFs in human cells after adenoviral introduction of TRF2-DN (Supplementary Fig. 1A), a dominant-negative allele of TRF2 (van Steensel et al. 1998; Karlseder et al. 1999). In contrast, MDC1 was not localized at chromosome ends in cells with telomere protection (Fig. 1A; Supplementary Fig. 1A). Furthermore, telomeric DNA was not recovered in 
A

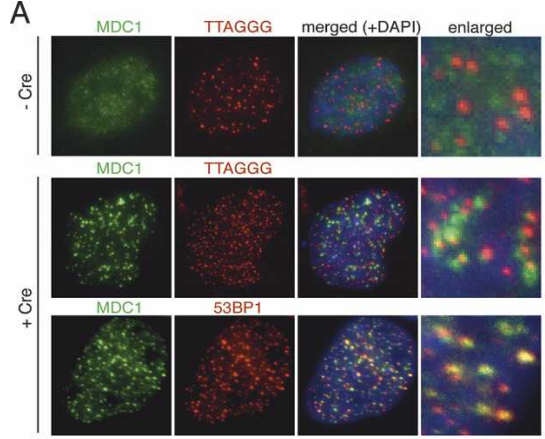

B
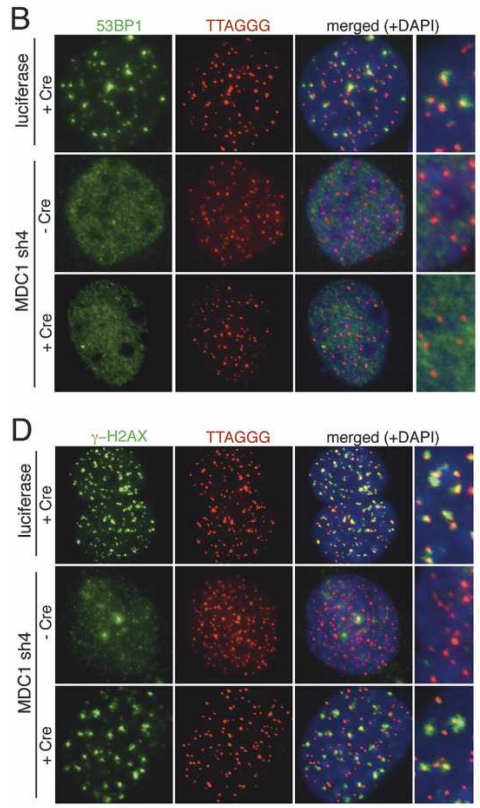

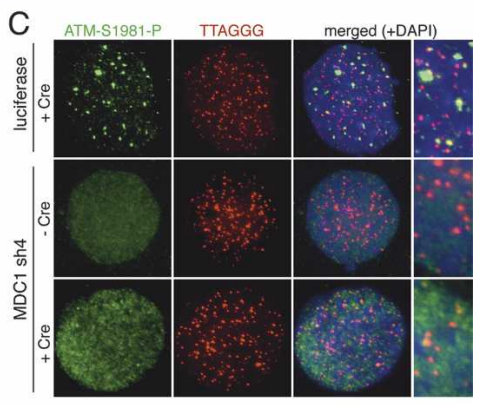

$\mathrm{E}$

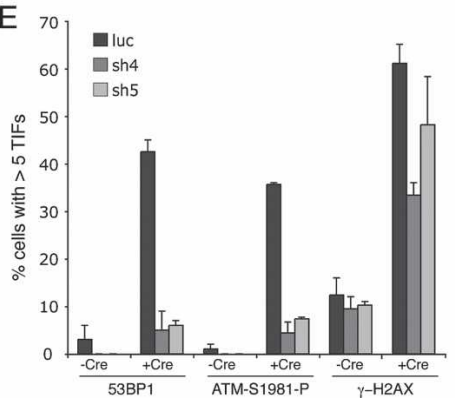

Figure 1. MDC1 localizes at dysfunctional telomeres and promotes the formation of TIFs. (A) MDC1 recruitment to chromosome ends after deletion of a conditional TRF2 allele in TRF2F/- lig4 $4^{-/-}$p53 $3^{-/-}$MEFs. Cells untreated or treated with Cre were fixed $72 \mathrm{~h}$ post-infection and processed for IF-FISH (MDC1 [green] costained with telomeric TTAGGG-specific FISH probe [red]) or IF (MDC1 [green] costained with 53BP1 [red]). (B-D) Effect of MDC1 knockdown on 53BP1 (B), ATM-S1981-P

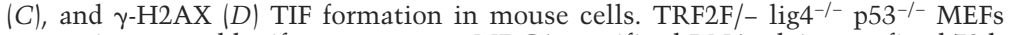
expressing control luciferase or mouse MDC1-specific shRNA, sh4, were fixed $72 \mathrm{~h}$ after mock or Cre infection and processed for IF-FISH (53BP1, ATM-S1981-P, and $\gamma$-H2AX [green]; TTAGGG probe [red]; and DNA counterstained with DAPI [blue]). (E) Bar graph of the frequency of TIF formation. At least 100 cells were scored for five or more telomeric 53BP1, ATM-S1981-P, and $\gamma$-H2AX foci in two independent experiments.

chromatin immunoprecipitation (ChIP) on cells expressing Flag-tagged MDC1 (Supplementary Fig. 1B). MDC1 binds to the Mre11/Rad50/Nbs1 complex (Goldberg et al. 2003), which associates with TRF2 and is present at functional human telomeres (Zhu et al. 2000). However, we found no indication that MDC1 contributed to the association of the Mre11 complex with TRF2. No association between MDC1 and TRF2 or other shelterin components was detected in immunoprecipitations (IPs) of endogenous proteins or overexpressed proteins (Supplementary Fig. 1C,D; data not shown), and MDC1 knockdown did not affect the association of Mre11 with TRF2 (Supplementary Fig. 1E). These data argue that MDC1 is not a constitutive component of functional telomeres but accumulates at telomeres that have been rendered dysfunctional through inhibition of TRF2.
To examine the role of MDC1 in the telomere damage response, we targeted $\mathrm{MDC1}$ with short hairpin RNAs (shRNAs). Immunofluorescence (IF) indicated a significant reduction in mouse MDC1 levels after treatment with two independent shRNAs, sh4 and sh5 (Supplementary Fig. 2A), and RT-PCR indicated a $75 \%-90 \%$ decrease in the MDC1 mRNA levels (Supplementary Fig. 2B). As expected, knockdown of mouse MDC1 abrogated the recruitment of 53BP1 and ATMS1981-P to dysfunctional mouse telomeres (Fig. 1B,C). In the shRNA-treated cells, 53BP1 and ATM-S1981-P remained diffuse and homogenous, while in control cells, both localized to telomeres and formed bright foci. Quantification of the TIF response indicated that MDC1 knockdown led to a sevenfold to 10 -fold reduction in the frequency of cells with more than five 53BP1 or ATM-S1981-P foci at dysfunctional telomeres (Fig. 1E). TIF formation by $\gamma$-H2AX was affected to a lesser extent by MDC1 knockdown (Fig. 1D,E) showing a reduction from $\sim 60 \%$ to $\sim 40 \%$ in the fraction of cells that were $\gamma$-H2AX TIF positive and there was a reduction in the size and/or intensity of the $\gamma$-H2AX foci. The same results were obtained with down-regulation of human MDC1 with two independent shRNAs in primary and transformed human cells (Supplementary Fig. 3A). MDC1 knockdown resulted in diminished TIF formation by 53BP1, ATMS1981-P, and Nbs1-S343-P, accompanied by a more modest effect on $\gamma$-H2AX (Supplementary Fig. 3B; data not shown). These results were further confirmed though inhibition of MDC1 with a dominant-negative allele, MDC1-FHA (Goldberg et al. 2003; Xu and Stern 2003). Overexpression of MDC1-FHA inhibited 53BP1 TIF formation in normal human fibroblasts as well as in A-T (ATM-deficient) cells and in Seckel syndrome cells, which are hypomorphic for ATR (Supplementary Fig. 4; O'Driscoll et al. 2003). Collectively, these data indicate that MDC1 promotes the formation of foci containing ATM-S1981-P, 53BP1, Nbs1, and $\gamma-\mathrm{H} 2 \mathrm{AX}$ at dysfunctional telomeres, as it does at DSBs.

Dysfunctional telomeres undergo end-toend fusions that are mediated by the NHEJ factors DNA ligase IV and Ku70 (Smogorzewska et al. 2002; Celli and de Lange 2005; Celli et al. 2006). This process can be quantified in metaphase spreads using telomeric DNA FISH (fluorescent in situ hybridization). In mouse and human cells, chromosomes typically fuse at a frequency of $0.2-0.3$ fusions per chromosome end within 72 $\mathrm{h}$ after inhibition of TRF2 (Fig. 2A; Supplementary Fig. 5A), and in TRF2-deficient mouse cells, the frequency of fusions increases to more than one fusion per chromosome after $5 \mathrm{~d}$, resulting in long trains of joined chromosomes (Celli and de Lange 2005). Most of the metaphases of MDC1 shRNA-treated mouse cells, however, did not contain joined chromosomes at $72 \mathrm{~h}$ after TRF2 deletion; only a small fraction of the metaphases showed a moderate number of fusions. Quantification revealed that MDC1 inhibition led to a three- to fourfold decrease in 

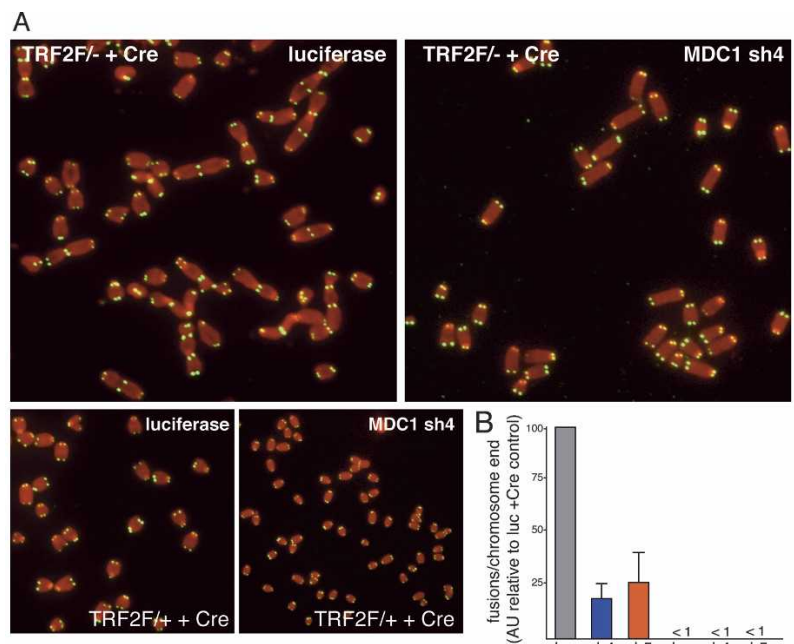

C native-ss [TTAGGG]n denatured - ds [TTAGGG]n
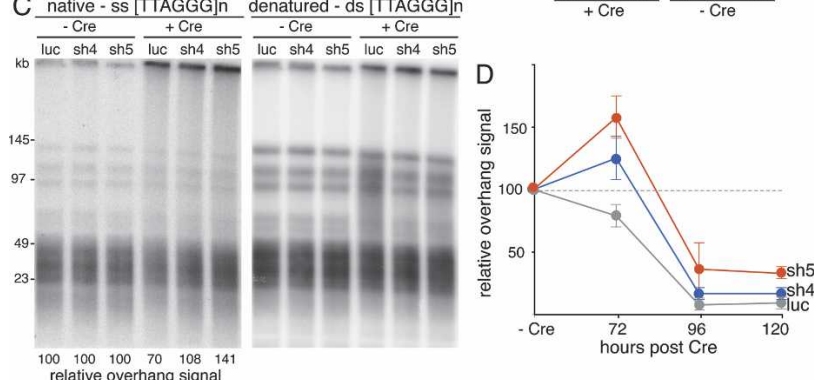

Figure 2. MDC1 stimulates NHEJ of dysfunctional telomeres. $(A)$ Metaphase spreads from TRF2F/- $553^{-/-}$and TRF2F $/+\mathrm{p} 53^{-/-}$MEFs expressing control luciferase or mouse MDC1-specific shRNA, sh4, $60 \mathrm{~h}$ after Cre infection. Telomeric signals were detected with FITCOO-(AATCCC $)_{3}$ oligonucleotide and are false-colored in green, DNA (DAPI) is false-colored in red. $(B)$ Quantification of telomere fusions in TRF2F/- p53 ${ }^{-/-}$MEFs, expressing the indicated shRNA mock- or Cre-infected for 60-72 h. Telomere fusions were counted as events per chromosome in metaphase spread as shown in $A$. Bar graph represents fusion frequency relative to control shRNA-treated cells in three independent experiments. $(C)$ Telomeric DNA analysis. (Left) In-gel assay detecting $3^{\prime}$ overhang of TRF2F/- p53 ${ }^{-/-}$MEFs expressing control luciferase or MDC1 shRNAs, sh4 and sh5. Cells were harvested $72 \mathrm{~h}$ post-infection with Cre and processed by in-gel hybridization to a $(\mathrm{CCCTAA})_{4}$ probe to detect ssTTAGGG repeats (native). (Right) The DNA was denatured in situ and rehybridized to the same probe to detect the total TTAGGG signal (denatured). Overhang signals were quantified with ImageQuant software and normalized to the total TTAGGG signal in the same lane. The numbers below the gel represent the percentage of normalized overhang signal compared with the normalized overhang signal for the same cells not treated with Cre. $(D)$ Quantification of telomeric overhang 72, 96, and $120 \mathrm{~h}$ post-infection with Cre in two independent experiments. The overhang signal at different time points after Cre infection is represented as a percentage of the overhang signal in the absence of Cre for the same cell line.

telomere fusions in mouse cells (Fig. 2A,B) and a similar decrease in human cells (Supplementary Fig. 5A,B). In TRF2F/- p53 $3^{-/-}$mouse cells the effect of MDC1 inhibition was most prominent at the early time points of telomere deprotection (60-72 $\mathrm{h}$ after introduction of Cre). At later time points, most chromosomes were fused into long trains both in control cells and in MDC1 shRNAtreated cells (data not shown). The NHEJ of dysfunctional telomeres was also affected when the recruitment of MDC1 to chromatin was blocked by lowering the amount of its binding partner, $\gamma-\mathrm{H} 2 \mathrm{AX}$, with shRNA to $\mathrm{H} 2 \mathrm{AX}$. A previously published shRNA resulted in effi- cient knockdown of H2AX in human cells (Fig. 3A) and resulted in abrogation of $\mathrm{MDC} 1$ foci at dysfunctional telomeres (Fig. 3B,C). These cells showed a threefold reduction in telomere fusions in response to inhibition of TRF2 (Fig. 3D,E), which is similar to the effect of MDC1 knockdown. These results indicate that $\gamma$-H2AX-mediated recruitment of MDC1 to chromatin accelerates the rate by which dysfunctional telomeres are repaired by NHEJ.

We next asked whether MDC1 affected the removal of the telomeric 3' overhang, a step that may be specific to the joining of dysfunctional telomeres. Prior data have shown that the telomeric $3^{\prime}$ overhang disappears after TRF2 inhibition, but recent data on mouse cells documented that this overhang removal does not occur when
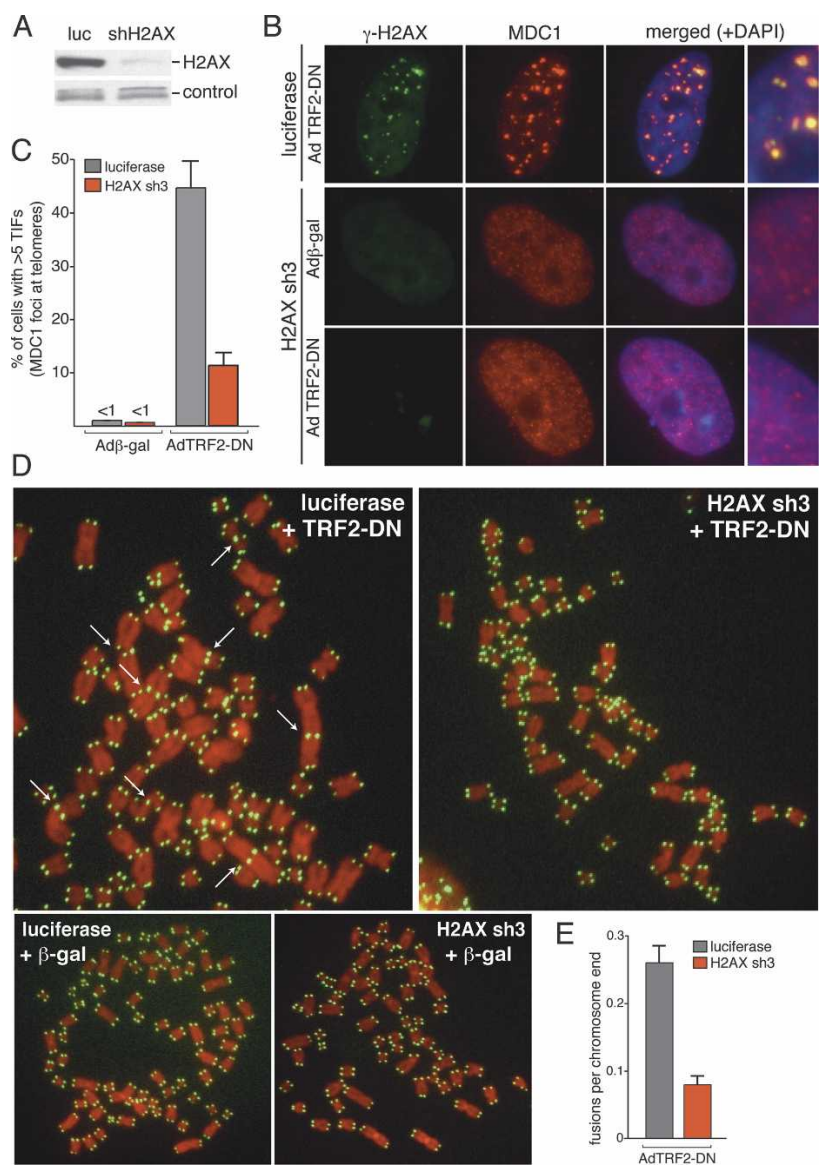

Figure 3. Recruitment of MDC1 to chromatin is required for effi cient NHEJ. (A) Immunoblot detection of H2AX in HeLa cells expressing control or H2AX-specific shRNA, sh3; nonspecific band from the same gel used as loading control. (B) Effect of H2AX knockdown on MDC1 TIF formation. HeLa cells expressing control luciferase or H2AX-specific shRNA were fixed 48 h post-infection with control ( $\beta$-gal) or TRF2-DN adenovirus and processed for IF ( $\gamma$-H2AX [green]; MDC1 [red]; and DNA counterstained with DAPI [blue]). (C) At least 100 cells were scored for five or more telomeric MDC1 foci in three independent experiments. (D) Metaphase spreads from HeLa cells expressing control luciferase or H2AX-specific shRNA 48 $\mathrm{h}$ post-infection with TRF2-DN or control ( $\beta$-gal) adenovirus. Telomeric signals were detected with FITC-OO-(AATCCC) ${ }_{3}$ oligonucleotide and are false-colored in green; DNA (DAPI) is false-colored in red. $(E)$ Quantification of telomere fusions in $C$ counted as events per chromosome end in three independent experiments. All the results were confirmed with a second independent $\mathrm{H} 2 \mathrm{AX}$ shRNA. 
the NHEJ pathway is blocked (Celli and de Lange 2005; Celli et al. 2006). In fact, when TRF2 is deleted from DNA ligase IV- or Ku70-deficient mouse cells, the overhang signals increase slightly, presumably due to degradation of the deprotected C-rich telomeric DNA strand. A similar increase in the overhang signal occurred when TRF2 was deleted from mouse cells lacking normal MDC1 levels (Fig. 2C,D). This effect coincided with the inhibition of NHEJ by MDC1 knockdown. At later time points, the overhang signal decreased (Fig. 2D) as expected from the occurrence of fusions at that stage. Thus, MDC1 knockdown affected the initial rate of overhang loss in mouse cells, but this result would be expected from a tight coupling between NHEJ and overhang processing reported previously (Celli and de Lange 2005; Celli et al. 2006). Further insight into the role of MDC1 in overhang processing was obtained in human cells. Previous work had shown that in human cells, overhang processing can occur before the actual joining of the telomeres, leading to the detection of telomeres with diminished overhangs that had not (yet) been joined (van Steensel et al. 1998). Thus, in human cells, overhang processing is not strictly coupled to NHEJ, as it is in mouse cells. This slight difference in the telomere fusion pathway in human and mouse cells allowed us to ask whether MDCl affected the overhang processing step itself. Although MDC1 clearly affected the rate of telomere fusion in transformed human cells (Supplementary Fig. 5A,B), there was no effect on overhang processing. After TRF2-DN expression, the same reduction in overhang signal $(\sim 40 \%)$ occurred in MDC1 knockdown and control cells (Supplementary Fig. 5C,D). This result argues against a role for $\mathrm{MDCl}$ in overhang processing per se and suggests that MDC1 promotes NHEJ of dysfunctional telomeres through another step.

We next asked whether the effect of MDC1 on the rate of NHEJ could be due to a change in cell cycle progression. It has not been firmly established whether the rate of NHEJ of dysfunctional mammalian telomeres is affected by the cell cycle stage (Smogorzewska et al. 2002). However, in fission yeast, telomere NHEJ is limited to G1 (Ferreira and Cooper 2001), and preferential NHEJ in G1 could explain why most telomere fusions in mouse cells are of the chromosome type (both sister telomeres fused). We therefore examined the effect of MDC1 knockdown on relevant cell cycle effectors that mediate the G1/S arrest after telomere dysfunction. MDC1 status affected neither the autophosphorylation of ATM nor the phosphorylation of Chk2 after TRF2 deletion from mouse cells (Fig. 4A). Furthermore, in human cells the up-regulation of p53 and p21 in response to TRF2-DN was unaltered by MDC1 knockdown (Fig. 4B), and primary human fibroblasts underwent senescence within a week of TRF2 inhibition regardless of the level of MDC1 (Fig. 4C; data not shown). Collectively, these data indicate that the activation of the G1/S checkpoint does not require the accumulation of $\mathrm{MDC1}$, 53BP1, ATM-S1981$\mathrm{P}$, or Nbs1-S343-P at the sites of telomere damage. Most likely, the ATM kinase is activated and retains the ability to phosphorylate its targets in the nucleoplasm despite the lack of stable association of ATM with the dysfunctional telomeres. The data suggested that the effect of MDC1 on NHEJ was unlikely to be due to altered effector pathways of the ATM signaling cascade. The results obtained with MDC1 knockdown in mouse cells also argued against the possibility that MDC1 affects

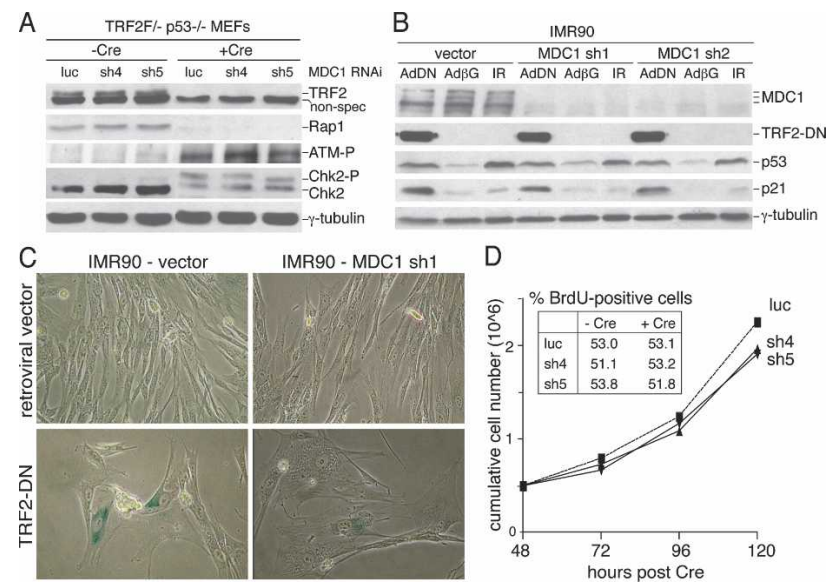

Figure 4. MDC1 knockdown does not affect checkpoint signaling in response to telomere dysfunction. (A) Immunoblot detection of ATM-S1981-P and Chk2-P after telomere deprotection. Whole-cell lysates of TRF2F/- lig4 $4^{-/-}$p53 $3^{-/-}$MEFs expressing control luciferase or MDC1-specific shRNAs, sh4 and sh5, were analyzed $72 \mathrm{~h}$ after mock or Cre infection. TRF2 and Rap1 immunoblots confirm efficient TRF2 deletion. (B) Detection of up-regulation of p53 and p21 in primary cells. Vector or MDC1 shRNA-treated IMR90 cells were harvested for immunoblot analysis $48 \mathrm{~h}$ post-infection with TRF2$\mathrm{DN}(\mathrm{AdDN})$, the $\beta$-gal adenovirus control (Ad $\beta G)$, or $1 \mathrm{~h}$ post-irradiation with 5 Gy (IR). The MDC1 immunoblot confirmed knockdown of MDC1 by two independent shRNAs; TRF2-DN expression is detected with a myc antibody. (C) Microscopic images of IMR90 cells, expressing vector or MDC1 shRNA, sh1, infected with empty vector control or TRF2-DN retrovirus stained for SA- $\beta$-galactosidase activity at $12 \mathrm{~d}$ after infection. (D) Lack of effect of MDC1 shRNA on proliferation of MEFs. Growth curve of TRF2F/- p53-/- MEFs after Cre infection. Inset table represents percent of BrdU-positive cells $72 \mathrm{~h}$ after mock or Cre infection.

NHEJ in a manner that involves cell cycle progression since these cells lacked a functional p53 pathway and their proliferation and BrdU uptake was not affected by MDC1 knockdown (Fig. 4D).

(We noted that upon more prolonged culturing [ $>2 \mathrm{wk}$ ], primary human fibroblasts expressing MDC1 shRNAs sh1 or sh2 started proliferating more slowly than the control cells and attained a senescence phenotype, even in the absence of TRF2-DN [Supplementary Fig. 6A,B; data not shown]. A senescence response to MDC1 knockdown is consistent with the diminished proliferation and senescent phenotype of MDC1 knockout MEFs [Lou et al. 2006]. However, a third human MDC1 shRNA [sh3] that down-regulated MDC1 efficiently and has been used in previous studies [Stewart et al. 2003; Lukas et al. 2004; Bekker-Jensen et al. 2005, 2006] did not have this senescence phenotype. Unexpectedly, we found that this shRNA [sh3] has extensive sequence identity to the mRNA for the ATM kinase and induced a significant reduction in ATM protein levels [Supplementary Fig. 6C,D]. Thus, MDC1 sh3 has a remarkably pathway-specific off-target effect whereby it affects both MDC1 itself and ATM, the main kinase responsible for the generation of MDC1-binding sites in damaged chromatin.)

Finally, we asked whether the effect of MDC1 on the NHEJ of dysfunctional telomeres could be explained by the diminished recruitment of 53BP1 or the Mre $11 \mathrm{com}$ plex to sites of telomere damage. Both 53BP1 and Mre11 were knocked down efficiently in human cells using previously reported shRNAs (Supplementary Fig. 7A,B). Despite this, the cells showed the same frequency of 
telomere fusions as the controls (Supplementary Fig. 7C,D).

The data argue that MDC1 is required to support the physiological pace of NHEJ-mediated repair of dysfunctional telomeres. We were unable to demonstrate an effect of MDC1 in the context of irradiation-induced DSBs using the Fraction of activity released (FAR) assay to monitor repair (Supplementary Fig. 8A,B) even though this assay showed the expected reduction in DSB repair when Phospho-3-kinase-related protein kinase (PIKK) inhibitors were applied (Supplementary Fig. 8C). Given that the FAR assay requires $\sim 10$ times more sites of DNA damage than are created through inhibition of TRF2, it may probe DSB repair in a range of DNA damage that is less dependent on MDC1. Thus, we have no reason to assume that the role of MDCl in NHEJ is specific for telomere dysfunction. On the contrary, our conclusions are consistent with the lower rate of plasmid transfection in cells overexpressing a dominant-negative allele of MDC1 (Stucki et al. 2005), the modest class switch defect of $\mathrm{MDC1}^{-/-}$mice, and their hypersensitivity to DSBs (Lou et al. 2006). In contrast, $\mathrm{MDC1}^{-/-}$mice have no overt defect in $\mathrm{V}(\mathrm{D}) \mathrm{J}$ recombination, an established measure of NHEJ (Lou et al. 2006). Therefore, it was unclear whether MDC1 promotes DSB repair through an effect on NHEJ or through its effect on cell cycle progression. This issue is clarified by our data since MDC1 affected NHEJ in the absence of a change in the cell cycle effectors of the ATM signaling pathway or discernable effects on cell cycle progression. We therefore conclude that MDC1 affects NHEJ directly. Its ability to accelerate NHEJ might be related to the interaction of MDC1 with DNA-PKcs/Ku (Lou et al. 2004). However, MDC1 also interacts with the homologous recombination (HR) protein Rad51 (Zhang et al. 2005), and the relevance of MDC1's interaction with these repair proteins remains to be established. Further use of telomere dysfunction to dissect the role of MDC1 in NHEJ might be helpful in this regard.

\section{Materials and methods}

shRNA knockdown

Phoenix cells were used to produce shRNA expressing pSUPERIOR retroviruses (OligoEngine). Recipient cells were infected five times every 12 $\mathrm{h}$ and selected in puromycin. Target sequences were luciferase, $5^{\prime}$-CG TACGCGGAATACTTCGA-3' (Dharmacon); hMDC1 sh1, 5'-GCAG AAGCCAATCAGCAAA-3'; hMDC1 sh2, 5'-AGAGGGACAATGATA CAAA-3'; hMDC1 sh 3, 5'-GTCTCCCAGAAGACAGTGA-3' (Stewart et al. 2003); mMDC1 sh4, 5'-ACAGCATGCAGTAATTGAA-3'; mMDC1 sh5, 5'-ACACAGCCGTTCTGTCTAA-3'. Published targets were used for Mre11.5 and Mre6 (Pichierri and Rosselli 2004); 53BP1.1 and 53BP2 (Wang et al. 2002), and H2AX-3 (Lukas et al. 2004). RT-PCR for mouse MDC1 was performed using oligo-dT ThermoScript RT-PCR system (Invitrogen) with $3 \mu \mathrm{g}$ RNA (Qiagen RNAeasy) and the following primers: forward, 5'-CTGTCCCTGAACTGGCTGTACCAG-3'; reverse, 5'-GG TAGATGACATTTCCAAATTGGA-3'. GAPDH (forward, 5' -TGAAGG TCGGTGTGAACGGATTTGGC-3'; reverse, 5' -CATGTAGGCCATGA GGTCCACCAC-3') served as a control.

Inhibition of TRF2

TRF2-DN and control $\beta$-galactosidase adenovirus (Karlseder et al. 1999) were used at 100 plaque-forming units (pfu) per cell for HeLa and IMR90 cells as previously described (Takai et al. 2003). Cre-mediated TRF2 deletion in mouse cells was performed by retroviral introduction of Cre recombinase into TRF2F/- p53 $3^{-/-}, \mathrm{TRF} 2 \mathrm{~F} /+\mathrm{p} 53^{-/-}$, and TRF2F/- lig $4^{-/-}$ p53 ${ }^{-/-}$MEFs (Celli and de Lange 2005). TRF2 deletion was not affected by RNA interference (RNAi) to MDC1.
IF and IF-FISH

Cells grown on coverslips were fixed for $10 \mathrm{~min}$ in $2 \%$ paraformaldehyde at room temperature and permeabilized for $5 \mathrm{~min}$ in $0.5 \%$ NP-40. Cells were blocked $30 \mathrm{~min}$ in PBG $(0.2 \%$ [w/v] cold water fish gelatin [Sigma G-17765]; $0.5 \%$ BSA in PBS) and incubated for $2 \mathrm{~h}$ at room temperature with the following antibodies in PBG: MDC1 (ab11169, Abcam); mMDC1 MAb (J. Chen, Mayo Clinic, Rochester, MN); rabbit $\alpha$-hTRF1, 371; 53BP1 MAb (T. Halazonetis, Wistar Institute, Philadelphia, PA); 53BP1 (100-304, Novus Biologicals); phospho-ATM-Ser 1981 (Cell Signaling); $\gamma$-H2AX MAb (Upstate Biotechnology); phospho-Nbs1 Ser-343 (Novus Biologicals). Secondary antibodies raised against mouse and rabbit were labeled with Alexa 488 (Molecular Probes) and Rhodamine Red-X (RRX, Jackson) respectively. IF-FISH was performed as described (Herbig et al. 2004) using the same primary and secondary antibodies as above using a Tamra-(TTAGGG) 3 PNA telomere probe (Applied Biosystems). DNA was counterstained with 4.6-diamidino-2-phenylindole (DAPI) and slides were mounted in $90 \%$ glycerol/10\% PBS containing 1 $\mu \mathrm{g} / \mathrm{mL}$ p-phenylene diamine (Sigma). Digital images were captured with a Zeiss Axioplan II microscope with a Hamamatsu C4742-95 camera using Improvision OpenLab software.

\section{Western blot analysis}

Antibodies used for immunoblotting were as follows: MDC1 (ab11169, Abcam); 53BP1 (100-305, Novus Biologicals); rabbit $\alpha$-Mre11, 874 (Zhu et al. 2000); H2AX (ab11175, Abcam); p53 (DO-1, Santa Cruz Biotechnology); p21 (F-5, Santa Cruz Biotechnology); rabbit $\alpha$-TRF2, 647 (Zhu et al. 2000); rabbit $\alpha$-mRap1, 1252 (Celli and de Lange 2005); Chk2 (BD Biosciences); myc (9E10, Oncogene/EMD Biosciences); $\gamma$-tubulin (clone GTU 488, Sigma).

TelomereFISH

FISH on metaphase spreads was performed as described previously (van Steensel et al. 1998) with a FITC-OO-(AATCCC) ${ }_{3}$ PNA telomere probe (Applied Biosystems).

Senescence-associated $\beta$-galactosidase (SA- $\beta$-gal) staining SA- $\beta$-gal staining on IMR90 cells infected with a TRF2-DN retrovirus $\left(\mathrm{pWZL}^{-T R F} 2^{\mathrm{AB} \Delta \mathrm{M}}\right.$ ) was performed as described previously (Dimri et al. 1995). Cells were kept under selection for $10 \mathrm{~d}$, replated, and stained $2 \mathrm{~d}$ later.

Pulse-field gel electrophoresis and in-gel detection of telomeric DNA Pulse-field gel electrophoresis and in-gel detection of mouse telomeric DNA were done as previously described (Celli and de Lange 2005). DNA (in plugs) was separated on a $1 \%$ agarose/0.5× TBE gel and ran for $24 \mathrm{~h}$ using CHEF-DRII PFGE apparatus (Bio-Rad) in $0.5 \times$ TBE running buffer (initial pulse, $5 \mathrm{sec}$; final pulse, $5 \mathrm{sec} ; 6 \mathrm{~V} / \mathrm{cm}^{2}$; at $14^{\circ} \mathrm{C}$ ). The native gel was hybridized with a ${ }^{32} \mathrm{P}-\gamma \mathrm{ATP}-$ labeled $(\mathrm{CCCTAA})_{4}$ oligonucleotide and then denatured and rehybridized. Gels were exposed onto a PhosphorImager screen overnight, and the single-stranded and total G-strand signal was quantified with ImageQuant software. The G-overhang signal was normalized to the total telomeric DNA and this normalized value was compared between samples.

BrdU incorporation

Cells grown on coverslips were incubated for $1 \mathrm{~h}$ in $10 \mu \mathrm{M} \mathrm{BrdU}$ at $37^{\circ} \mathrm{C}$; fixed in $75 \%$ ethanol, $25 \% 0.05 \mathrm{M}$ glycine $(\mathrm{pH} 2.2)$ for $45 \mathrm{~min}$ at $-20^{\circ} \mathrm{C}$; washed twice in PBS; and processed for IF using $\alpha$-BrdU-FITC-conjugated antibody (BD Biosciences).

\section{Acknowledgments}

We are grateful to David Chen, Junjie Chen, Steve Elledge, Thanos Halazonetis, and David Stern for generous gifts of reagents. Dirk Hockemeyer provided help and advice and Akimitsu Konishi and Hiro Takai are thanked for critical comments on this manuscript. N.D. is supported by a Howard Hughes Predoctoral Fellowship. This work was supported by grants from the NIH (GM49046 and AG16642). 


\section{References}

Bekker-Jensen, S., Lukas, C., Melander, F., Bartek, J., and Lukas, J. 2005. Dynamic assembly and sustained retention of 53BP1 at the sites of DNA damage are controlled by Mdc1/NFBD1. J. Cell Biol. 170: 201211.

Bekker-Jensen, S., Lukas, C., Kitagawa, R., Melander, F., Kastan, M.B. Bartek, J., and Lukas, J. 2006. Spatial organization of the mammalian genome surveillance machinery in response to DNA strand breaks. J. Cell Biol. 173: 195-206.

Celli, G. and de Lange, T. 2005. DNA processing not required for ATMmediated telomere damage response after TRF2 deletion. Nat. Cell Biol. 7: 712-718.

Celli, G.B., Lazzerini Denchi, E., and de Lange, T. 2006. Ku70 stimulates fusion of dysfunctional telomeres yet protects chromosome ends from homologous recombination. Nat. Cell Biol. 8: 885-890.

d'Adda di Fagagna, F., Reaper, P.M., Clay-Farrace, L., Fiegler, H., Carr, P., Von Zglinicki, T., Saretzki, G., Carter, N.P., and Jackson, S.P. 2003. A DNA damage checkpoint response in telomere-initiated senescence. Nature 426: 194-198.

de Lange, T. 2005. Shelterin: The protein complex that shapes and safeguards human telomeres. Genes \& Dev. 19: 2100-2110.

Dimri, G.P., Lee, X., Basile, G., Acosta, M., Scott, G., Roskelley, C., Medrano, E.E., Linskens, M., Rubelj, I., Pereira-Smith, O., et al. 1995. A biomarker that identifies senescent human cells in culture and in aging skin in vivo. Proc. Natl. Acad. Sci. 92: 9363-9367.

Ferreira, M.G. and Cooper, J.P. 2001. The fission yeast Tazl protein protects chromosomes from Ku-dependent end-to-end fusions. Mol. Cell 7: 55-63.

Goldberg, M., Stucki, M., Falck, J., D'Amours, D., Rahman, D., Pappin, D., Bartek, J., and Jackson, S.P. 2003. MDC1 is required for the intraS-phase DNA damage checkpoint. Nature 421: 952-956.

Herbig, U., Jobling, W.A., Chen, B.P., Chen, D.J., and Sedivy, J.M. 2004. Telomere shortening triggers senescence of human cells through a pathway involving ATM, p53, and p21(CIP1), but not p16(INK4a). Mol. Cell 14: 501-513.

Karlseder, J., Broccoli, D., Dai, Y., Hardy, S., and de Lange, T. 1999. p53and ATM-dependent apoptosis induced by telomeres lacking TRF2. Science 283: 1321-1325.

Lee, M.S., Edwards, R.A., Thede, G.L., and Glover, J.N. 2005. Structure of the BRCT repeat domain of MDC1 and its specificity for the free $\mathrm{COOH}$-terminal end of the $\gamma-\mathrm{H} 2 \mathrm{AX}$ histone tail. J. Biol. Chem. 280: 32053-32056.

Lou, Z., Chen, B.P., Asaithamby, A., Minter-Dykhouse, K., Chen, D.J., and Chen, J. 2004. MDC1 regulates DNA-PK autophosphorylation in response to DNA damage. J. Biol. Chem. 279: 46359-46362.

Lou, Z., Minter-Dykhouse, K., Franco, S., Gostissa, M., Rivera, M.A., Celeste, A., Manis, J.P., van Deursen, J., Nussenzweig, A., Paull, T.T., et al. 2006. MDC1 maintains genomic stability by participating in the amplification of ATM-dependent DNA damage signals. Mol. Cell 21: $187-200$

Lukas, C., Melander, F., Stucki, M., Falck, J., Bekker-Jensen, S., Goldberg, M., Lerenthal, Y., Jackson, S.P., Bartek, J., and Lukas, J. 2004. Mdc1 couples DNA double-strand break recognition by Nbs1 with its H2AX-dependent chromatin retention. EMBO J. 23: 2674-2683.

O’Driscoll, M., Ruiz-Perez, V.L., Woods, C.G., Jeggo, P.A., and Goodship, J.A. 2003. A splicing mutation affecting expression of ataxia-telangiectasia and Rad3-related protein (ATR) results in Seckel syndrome. Nat. Genet. 33: 497-501.

Pichierri, P. and Rosselli, F. 2004. The DNA crosslink-induced S-phase checkpoint depends on ATR-CHK1 and ATR-NBS1-FANCD2 pathways. EMBO J. 23: 1178-1187.

Rothkamm, K. and Lobrich, M. 2003. Evidence for a lack of DNA doublestrand break repair in human cells exposed to very low X-ray doses. Proc. Natl. Acad. Sci. 100: 5057-5062.

Smogorzewska, A. and de Lange, T. 2002. Different telomere damage signaling pathways in human and mouse cells. EMBO J. 21: 43384348.

Smogorzewska, A., Karlseder, J., Holtgreve-Grez, H., Jauch, A., and de Lange, T. 2002. DNA ligase IV-dependent NHEJ of deprotected mammalian telomeres in G1 and G2. Curr. Biol. 12: 1635-1644.

Stewart, G.S., Wang, B., Bignell, C.R., Taylor, A.M., and Elledge, S.J. 2003. MDC1 is a mediator of the mammalian DNA damage check- point. Nature 421: 961-966.

Stucki, M. and Jackson, S.P. 2006. $\gamma \mathrm{H} 2 \mathrm{AX}$ and MDC1: Anchoring the DNA-damage-response machinery to broken chromosomes. DNA Repair (Amst.) 5: 534-543.

Stucki, M., Clapperton, J.A., Mohammad, D., Yaffe, M.B., Smerdon, S.J., and Jackson, S.P. 2005. MDC1 directly binds phosphorylated histone $\mathrm{H} 2 \mathrm{AX}$ to regulate cellular responses to DNA double-strand breaks. Cell 123: 1213-1226.

Takai, H., Smogorzewska, A., and de Lange, T. 2003. DNA damage foci at dysfunctional telomeres. Curr. Biol. 13: 1549-1556.

van Steensel, B., Smogorzewska, A., and de Lange, T. 1998. TRF2 protects human telomeres from end-to-end fusions. Cell 92: 401-413.

Wang, B., Matsuoka, S., Carpenter, P.B., and Elledge, S.J. 2002. 53BP1, a mediator of the DNA damage checkpoint. Science 298: 1435-1438.

$\mathrm{Xu}, \mathrm{X}$. and Stern, D.F. 2003. NFBD1/MDC1 regulates ionizing radiationinduced focus formation by DNA checkpoint signaling and repair factors. FASEB J. 17: 1842-1848.

Zhang, J., Ma, Z., Treszezamsky, A., and Powell, S.N. 2005. MDC1 interacts with Rad51 and facilitates homologous recombination. Nat. Struct. Mol. Biol. 12: 902-909.

Zhu, X.D., Kuster, B., Mann, M., Petrini, J.H., and de Lange, T. 2000 Cell-cycle-regulated association of RAD50/MRE11/NBS1 with TRF2 and human telomeres. Nat. Genet. 25: 347-352. 


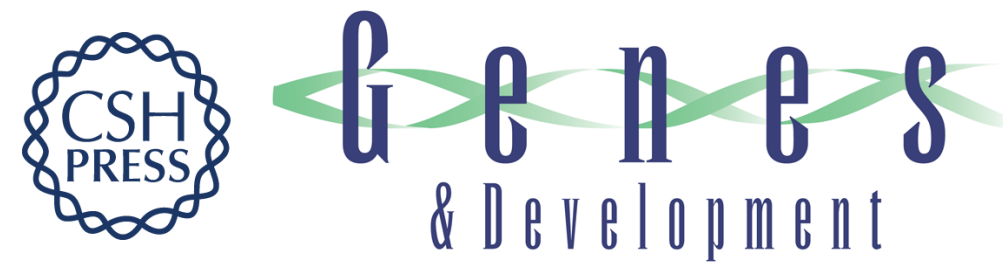

\section{MDC1 accelerates nonhomologous end-joining of dysfunctional telomeres}

Nadya Dimitrova and Titia de Lange

Genes Dev. 2006, 20:

Access the most recent version at doi:10.1101/gad.1496606

Supplemental http://genesdev.cshlp.org/content/suppl/2006/11/16/20.23.3238.DC1
Material

References This article cites 28 articles, 12 of which can be accessed free at: http://genesdev.cshlp.org/content/20/23/3238.full.html\#ref-list-1

License

Email Alerting Receive free email alerts when new articles cite this article - sign up in the box at the top Service right corner of the article or click here.

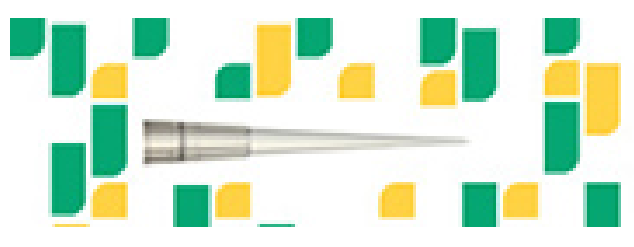

